\title{
Cerebral Hemorrhage, Splenic and Renal Embolisms Due to Infective Endocarditis
}

\author{
Zhou-Wen Chen, Li-Zheng Fang, Ling-Xiu Huang, Bo Zhou and Yong-Mei Han
}

\begin{abstract}
Infective endocarditis is occurring more and more frequently in elderly persons and it has been associated with various clinical, bacteriological, and prognostic features. We present a 63-year-old woman with infective endocarditis which is the first reported case with cerebral hemorrhage, splenic and renal embolisms.
\end{abstract}

Key words: cerebral hemorrhage, splenic embolism, renal embolism, endocarditis, degenerative valve disease

(Inter Med 48: 1247-1250, 2009)

(DOI: 10.2169/internalmedicine.48.2054)

\section{Introduction}

Infective endocarditis (IE) has continued to be an important disease with stable incidence and high morbidity and mortality in Asia (1). Elderly patients are emerging as a population at high risk for IE and diagnosis of IE in the elderly is often delayed, which directly results in multiorgan involvement (2). Cases of brain hemorrhage, splenic and renal embolisms from IE, as in the following report, have not been described previously.

\section{Case Report}

In November 2007, A 63-year-old woman was admitted to a local hospital with chills and fever $\left(38^{\circ} \mathrm{C}\right)$. Because of suspecting pharyngitis, the doctor prescribed oral penicillin for 5 days and the patient's temperature recovered to normal. Several days later, the patient relapsed with fever, leading to her first hospitalization. She was febrile $\left(\mathrm{T}_{\max } 40^{\circ} \mathrm{C}\right)$ during hospitalization: fever was persistent for 6 to 7 hours every day and then stopped after sweating. The laboratory findings at the beginning of admission were as follows: The leukocyte count was $8.0 / \mathrm{nL}$ (normal counts, 4.0 to $10.0 / \mathrm{nL}$ ), the hemoglobin level was $94 \mathrm{~g} / \mathrm{L}$ (normal levels, 110 to 150 $\mathrm{g} / \mathrm{L}$ ), the C-reactive protein level was $74 \mathrm{mg} / \mathrm{L}$ (normal, 0 to $5 \mathrm{mg} / \mathrm{L}$ ) and the blood sedimentation rate was $86 \mathrm{~mm} / \mathrm{h}$ (normal, 0 to $20 \mathrm{~mm} / \mathrm{h}$ ). Urine examination showed occult blood 2+. Abdominal sonography showed mild splenomegaly. The transthoracic echocardiogram documented moderate regurgitation of the aortic valve. Chest Xray showed a little effusion in the left pleural cavity. The patient was treated with levofloxacin for 7 days, but treatment failed. Piperacillin sodium was subsequently used for 10 days and the patient was discharged without fever. In February 2008, the patient was hospitalized with fever for the second time. On this occasion, the patient complained of dull pain of the left back and fatigue. Chest X-ray and computed tomography (CT) of the lung showed infiltrates in the left lung, which were interpreted as pneumonia. The patient was treated with a combination of cefazolin and gentamicin as empiric antibiotics for 2 weeks and fever subsided gradually. In May 2008, the patient was brought to the emergency unit of the same hospital with sudden onset of right side hemiplegia, headache and dizziness. CT of head showed hemorrhage of left parietal lobe (Fig. 1). The symptoms were relieved after expectant treatment. However, she developed a fever of $38.8^{\circ} \mathrm{C}$ and dull pain of left back about 4 days after admission. Despite antibiotic therapy, febrile temperatures and pain of left back persisted. During this hospitalization, the echocardiographic examination of the patient also documented moderate regurgitation of the aortic valve, but no vegetation was seen. The patient left hospital and took non-steroidal anti-inflammatory drug (NSAID) for fever and pain. More than 7 blood cultures, including aerobic and anaerobic blood cultures, had been performed during the previous three times of hospitalization and no significant results were found. No plasmodium was observed on blood 


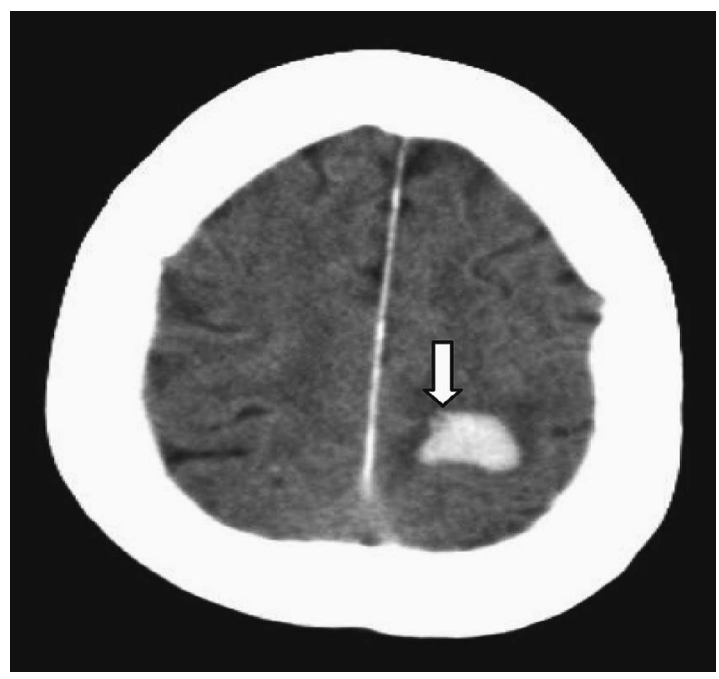

Figure 1. Brain CT obtained in May 2008. Arrow indicates the high density lesion of the left parietal lobe.

smear and bone marrow puncture examination revealed normal findings.

In October 2008, the patient was hospitalized for the fourth time, because of intermittent fever and pain of the left back. Associated symptoms included general malaise, anorexia, and the loss of $5 \mathrm{~kg}$ body weight within the previous a year. The patient denied any operation or surgical procedure.

On admission, the vital signs were as follows: blood pressure: 124/62 $\mathrm{mmHg}$; pulse rate: 90/min; respiratory rate: $23 /$ min; body temperature: $39.3^{\circ} \mathrm{C}$. Physical examination revealed respiratory harshness, a grade $2 / 6$ diastolic murmur over the left lower sternal border, and percussion tenderness over left kidney region. Clinical neurologic examination showed right limb myophagism, weakness of the right upper limb (grade 3) and right lower limb (grade 4), and rightsided hemihypesthesia. Four sets of blood cultures were collected on the first hospital day. The chest X-ray examination showed increased pulmonary markings, enlarged heart shadow and widened arota. The transthoracic and transesophageal echocardiogram documented moderate regurgitation and mild stenosis of the aortic valve. Abdominal CT showed multiple hypodense lesions of the spleen and kidney (Fig. 2), which was considered spleen and kidney infarction. Cerebral CT indicated left parietal lobe softening lesion. Complementary blood tests revealed the following: hemoglobin $89 \mathrm{~g} / \mathrm{L}$; leukocyte count $11.4 / \mathrm{nL}$ with $84.7 \%$ neutrophils. Erythrocyte sedimentation rate was $72 \mathrm{~mm} / \mathrm{h}$; and the occult blood test of urine was $3+$. Serologic tests for lupus anticoagulant, anticardiolipin antibodies, ANA, anti-dsDNA, ANCA, HBV and HIV serology were all negative. Rheumatoid factor was positive. On the third day at the hospital, Streptococcus anginosus grew in four blood cultures.

Ultimately, definite subacute IE was diagnosed according to the Duke criteria, including four blood culture results of Streptococcus anginosus; embolism of multiple organs; and

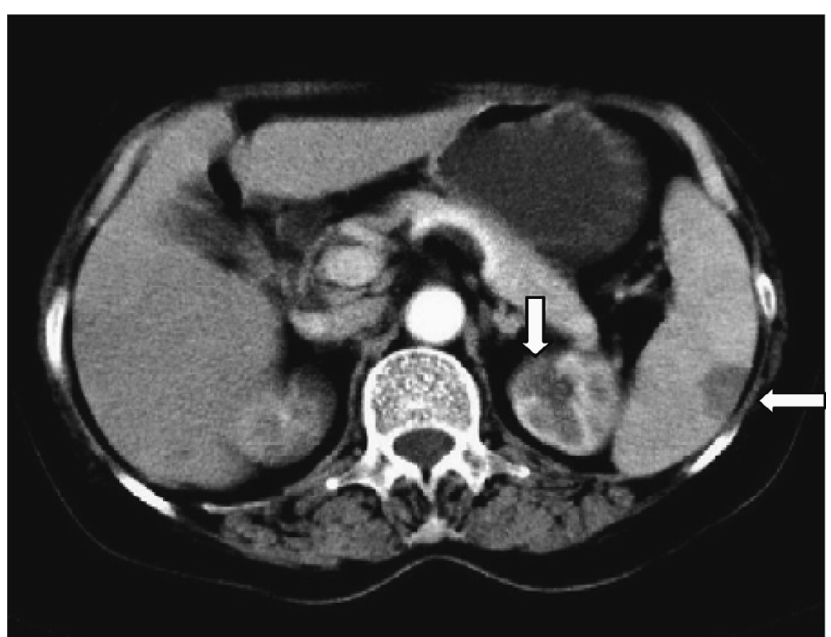

Figure 2. Abdominal CT obtained in October 2008. Arrows indicate hypodense lesions of the spleen and kidney.

such clinical and laboratory features as fever, heart murmur, anemia and positive rheumatoid factor. The patient was given penicillin (12 million units daily) and gentamicin (80 $\mathrm{mg} / 8 \mathrm{hr}$ ) for a total of 35 days. She was discharged after a total of 42 days of hospitalization. The patient was well after one month of follow-up.

\section{Discussion}

The incidence of IE does not appear to have declined in recent years, and the increased life span renders elderly people an increasing proportion of patients with IE (3). Different from the IE based on rheumatic heart disease and congenital heart disease in the younger population, more and more elderly patients develop IE as a result of degenerative valve disease. The present case is consistent with the recognition that elderly individuals with degenerative valvular disease are the most vulnerable population (4).

IE is an uncommon clinical entity that, if unrecognized, leads to serious morbidity and mortality (6). In a large prospective study, increasing age emerges as a major determinant of the clinical characteristics of IE and elderly patients were reported to more frequently undergo hospitalization or an invasive procedure before IE onset (6). Previous reports also showed that IE in advanced age was associated with a more severe prognosis $(7,8)$ and with a high occurrence of complications (9). The present case went misdiagnosed for a year. The patient carried a poor prognosis because of arterial embolism of multiple organs and cerebral hemorrhage.

The clinical features of the present patient's case illustrate a few interesting points. The endocarditis was afebrile and insidious when the patient was brought to the emergency unit of the local hospital with cerebral hemorrhage. Cerebral hemorrhage is often seen in the elderly with arteriosclerosis, hypertension, diabetes mellitus, etc. It may also be caused by IE, which occurs when the infected aneurysm induced by bacterial embolus ruptures. Cerebral hemorrhage of the pre- 
sent patient had ever been considered the result of arteriosclerosis and hence the etiology of recurrent fever was not identified. It illustrates that a high index of suspicion is necessary for endocarditis in cases presenting with a stroke with an unknown etiology, even without the common IE symptoms.

Positive blood cultures remain the cornerstone of diagnosis and provide live bacteria for susceptibility testing. However, blood cultures are negative in $2.5-31 \%$ of all cases of IE, which are associated with a delay in diagnosis $(10,11)$. Negative cultures arise most commonly as a consequence of prior antibiotic administration. A report showed that $4 / 5$ culture-negative cases had documented use of antibiotics prior to hospital admission (12). In the present case, more than 7 blood cultures were negative during the previous three times of hospitalization because of a history of antibiotic treatment. After her hospitalization in our hospital, the four blood cultures had all been taken before antibiotic administration, and the blood culture results of Streptococcus anginosus further confirmed our diagnosis. Thus, a negative result of blood culture cannot rule out the diagnosis of infective endocarditis.

Echocardiography plays a key role in the diagnosis and management of IE $(13,14)$. Early use of transthoracic echocardiogram (TTE) may allow a rapid diagnosis of IE (15), but care must be taken to avoid over-reliance on this technique. For clinically suspected cases in a survey, TTE had a sensitivity, specificity and negative predictive value of $71 \%$, $98 \%$ and $99 \%$, respectively but a positive predictive value of only $57 \%$, indicating that based on the TTE result alone,
$43 \%$ of patients would have been wrongly diagnosed as genuine IE cases (12). Werner et al showed that the detection rate of vegetations by TTE was lower in older patients (16). The echocardiographic examination of the present patient documented moderate regurgitation with mild stenosis of the aortic valve, but no vegetation was seen. These findings suggest, in keeping with those of other studies $(17,18)$, that echocardiogram alone is not an ideal method by which to diagnose IE.

Clinical features at presentation continue to be important for the diagnosis of IE (12). In the present case, definite subacute IE was diagnosed according to the features, including four blood culture results of Streptococcus anginosus; embolism of multiple organs; and such clinical and laboratory features as fever, heart murmur, anemia and positive rheumatoid factor. The features were used to guide investigation and treatment of IE prior to echocardiography, or when transthoracic echocardiogram is negative. If endocarditis had been diagnosed earlier and if infection had been treated appropriately, embolic complications might have been prevented, and a more favorable outcome might have been achieved.

In summary, several lessons for the future management of infective endocarditis in the elderly can be drawn: one needs to have a high index of suspicion for endocarditis in elderly patients. Generally, in the case with high fever of unknown origin, clinicians should not immediately begin antibiotics. When dealing with a stroke with an unclear etiology, clinicians should consider the existence of a concealed neoplasia with stroke as its first manifestation.

\section{References}

1. Yiu KH, Siu CW, Lee KL, et al. Emerging trends of community acquired infective endocarditis. Int J Cardiol 121: 119-122, 2007.

2. Wang JL, Hung CC, Hsieh SM, Chang SC. Clinical features and outcome analysis of infective endocarditis in elderly patients. J Formos Med Assoc 103: 416-421, 2004.

3. Moreillon P, Que YA. Infective endocarditis. Lancet 363: 9403, 2004.

4. Hill EE, Herijgers P, Herregods MC, Peetermans WE. Evolving trends in infective endocarditis. Clin Microbiol Infect 12: 5-12, 2006.

5. Paterick TE, Paterick TJ, Nishimura RA, Steckelberg JM. Complexity and subtlety of infective endocarditis. Mayo Clin Proc 82: 615-621, 2007.

6. Durante-Mangoni E, Bradley S, Selton-Suty C, et al. Current features of infective endocarditis in elderly patients: results of the International Collaboration on Endocarditis Prospective Cohort Study. Arch Intern Med 168: 2095-2103, 2008.

7. Selton-Suty C, Hoen B, Grentzinger A, et al. Clinical and bacteriological characteristics of infective endocarditis in the elderly. Heart 77: 260-263, 1997.

8. Kupferwasser I, Darius H, Müller AM, et al. Clinical and morphological characteristics in Streptococcus bovis endocarditis: a comparison with other causative microorganism in 177 cases. Heart 80: 276-280, 1998.

9. Terpening MS, Buggy BP, Kauffman CA. Infective endocarditis: clinical features in young and elderly patients. Am J Med 83: 626634, 1987.
10. Naber CK, Erbel R. Diagnosis of culture negative endocarditis: novel strategies to prove the suspect guilty. Heart 89: 241-243, 2003.

11. Prendergast $\mathrm{BD}$. The changing face of infective endocarditis. Heart 92: 879-885, 2006.

12. Todd AJ, Leslie SJ, Macdougall M, Denvir MA. Clinical features remain important for the diagnosis of infective endocarditis in the modern era. Q J Med 99: 23-31, 2006.

13. Erbel R, Rohmann S, Drexler M, et al. Improved diagnostic value of echocardiography in patients with infective endocarditis by transoesophageal approach: a prospective study. Eur Heart J 9: 4353, 1988.

14. Habib G, Derumeaux G, Avierinos JF, et al. Value and limitations of the Duke criteria for the diagnosis of infective endocarditis. J Am Coll Cardiol 33: 2023-3029, 1999.

15. Kuruppu JC, Corretti M, Mackowiak P, Roghmann M-C. Overuse of transthoracic echocardiography in the diagnosis of native valve endocarditis. Arch Int Med 162: 1715-1720, 2002.

16. Werner GS, Schulz R, Fuchs JB, et al. Infective endocarditis in the elderly in the era of transesophageal echocardiography: clinical features and prognosis compared with younger patients. Am J Med 100: 90-97, 1996.

17. Jaffe WM, Morgan DE, Pearlman AS. Infective endocarditis, 1983-1988: echocardiographic findings and factors influencing morbidity and mortality. J Am Coll Cardiol 15: 1227-1233, 1990.

18. Mugge A, Daniel WG, Frank G. Echocardiography in infective endocarditis: reassessment of prognostic implications of vegetation 
Inter Med 48: 1247-1250, 2009 DOI: 10.2169/internalmedicine.48.2054

size determined by the transthoracic and the transesophageal ap-

proach. J Am Coll Cardiol 14: 631-638, 1989.

(C) 2009 The Japanese Society of Internal Medicine http://www.naika.or.jp/imindex.html 